Tohoku Math. J.

68 (2016), 457-469

\title{
RICHNESS OF SMITH EQUIVALENT MODULES FOR FINITE GAP OLIVER GROUPS
}

\author{
Dedicated to Professor Mikiya Masuda on his sixtieth birthday
}

Toshio SUMI

(Received February 25, 2014, revised January 29, 2015)

\begin{abstract}
Let $G$ be a finite group not of prime power order. Two real $G$-modules $U$ and $V$ are $\mathcal{P}(G)$-connectively Smith equivalent if there exists a homotopy sphere with smooth $G$-action such that the fixed point set by $P$ is connected for all Sylow subgroups $P$ of $G$, it has just two fixed points, and $U$ and $V$ are isomorphic to the tangential representations as real $G$-modules respectively. We study the $\mathcal{P}(G)$-connective Smith set for a finite Oliver group $G$ of the real representation ring consisting of all differences of $\mathcal{P}(G)$-connectively Smith equivalent $G$-modules, and determine this set for certain nonsolvable groups $G$.
\end{abstract}

Introduction. Let $G$ be a finite group. The Smith problem given in the footnote on [43, p. 406] is a fundamental problem in the theory of transformation groups. Let $\Sigma$ be a homotopy sphere with smooth $G$-action such that $\Sigma$ has just two fixed points, say $a$ and $b$. The Smith problem asks whether tangential representations $T_{a}(\Sigma)$ and $T_{b}(\Sigma)$ are isomorphic as real $G$-modules. Two real $G$-modules $U$ and $V$ are called Smith equivalent if there exists a smooth action of $G$ on the homotopy sphere $\Sigma$ such that $\Sigma^{G}=\{a, b\}, T_{a}(\Sigma) \cong U$ and $T_{b}(\Sigma) \cong V$ as real $G$-modules. If the action is semi-free, that is, the isotropy subgroup of any point different from $a$ and $b$ is the trivial group, Smith equivalent real modules are isomorphic $[24,1]$. On the other hand, it is well-known that there are infinitely many groups $G$ possessing nonisomorphic, Smith equivalent real $G$-modules (cf. [36, 37, 38, 39, 10, 12, 23, 45, 6, 7, $14,13])$. Let $\operatorname{Sm}(G)$ be the subset of $\operatorname{RO}(G)$, the real representation ring, consisting of the differences $[U]-[V]$ such that $U$ and $V$ are Smith equivalent. The representation sphere $S(V \oplus \mathbb{R})$ implies that $0=[V]-[V] \in \operatorname{Sm}(G)$. By Sanchez's result [41, (1.11)] and Smith's theorem [3, III (5.1)], Smith equivalent real $G$-modules have isomorphic restrictions to any cyclic subgroup with odd prime power order of $G$. Morimoto [25] showed that $\operatorname{Sm}(G)$ is a subset of $\mathrm{RO}(G)^{\{\cap 2(G)\}}$, the kernel of Fix : $\mathrm{RO}(G) \rightarrow \operatorname{RO}(G / \cap 2(G))$, where $\cap 2(G)$ denotes the normal subgroup of $G$ given as the intersection of all subgroups of $G$ with index 2 or 1 .

We consider a little bit stronger equivalence relation. Let $\mathcal{P}(G)$ be the set of all prime power order subgroups of $G$. Two real $G$-modules $U$ and $V$ are called $\mathcal{P}(G)$-connectively Smith equivalent if there exists a smooth action of $G$ on a homotopy sphere $S$ such that $S^{G}=\{a, b\}, T_{a}(S) \cong U$ and $T_{b}(S) \cong V$ as real $G$-modules, and $S^{P}$ is connected for any

2010 Mathematics Subject Classification. Primary 57S17; Secondary $20 \mathrm{C} 15$.

Key words and phrases. Smith problem, tangential representation, gap group, Oliver groups.

This work was partially supported by KAKENHI (24540083). 
subgroup $P \in \mathcal{P}(G)$. Let $\mathrm{PSm}^{c}(G)$ be the subset of $\mathrm{RO}(G)$ consisting of the differences $[U]-[V]$ such that $U$ and $V$ are $\mathcal{P}(G)$-connectively Smith equivalent. Note that $\operatorname{PSm}^{c}(G)$ is a subset of $\operatorname{Sm}(G)$ and that $0 \in \operatorname{PSm}^{c}(G)$ if and only if $G$ is not of prime power order. If $G$-modules $U$ and $V$ are $\mathcal{P}(G)$-connectively Smith equivalent, then for any subgroup $P$ of $G$ of prime power order, $\operatorname{dim} U^{P}$ is positive, which implies that $U$ contains an irreducible $G$-module $W$ with $\operatorname{dim} W^{P}>0$. If any irreducible $G$-module $W$ satisfies $\operatorname{dim} W^{P}>0$ for any subgroup $P$ of $G$ of prime power order, then Smith equivalent $G$-modules are $\mathcal{P}(G)$ connectively Smith equivalent and in particular, $\operatorname{PSm}^{c}(G)=\operatorname{Sm}(G)$.

An Oliver group is characterized as a finite group acting smoothly on a disk without fixed points [32, Theorem 7] or one acting smoothly on a sphere with just one fixed point [19, Theorem A]. In this article we study the $\mathcal{P}(G)$-connective $\operatorname{Smith} \operatorname{sets} \operatorname{PSm}^{c}(G)$ of various Oliver groups $G$. By the slice theorem and the Smith theory, $\operatorname{PSm}^{c}(G)$ is a subset of the kernel $\operatorname{RO}(G)_{\mathcal{P}(G)}$ of the homomorphism $\oplus_{P \in \mathcal{P}(G)} \operatorname{Res}_{P}^{G}: \operatorname{RO}(G) \rightarrow \oplus_{P \in \mathcal{P}(G)} \operatorname{RO}(P)$.

We remark that the Smith set $\operatorname{Sm}(G)$ is not a subset of $\operatorname{RO}(G)_{\mathcal{P}(G)}$ in general (cf. [5]) and the set $\operatorname{Sm}(G) \backslash \operatorname{RO}(G)_{\mathcal{P}(G)}$ is a finite set (cf. [2, 40, 29]). The set $\operatorname{Sm}(G) \backslash \operatorname{RO}(G)_{\mathcal{P}(G)}$ is not empty for any group possessing a cyclic quotient group of order 8 (cf. [5]), while it is empty if $G$ has no element of order 8 . Let $G^{\text {nil }}$ be the smallest normal subgroup of $G$ such that $G / G^{\text {nil }}$ is nilpotent. By definition, $G^{\text {nil }}$ is a subgroup of $\cap 2(G)$. A real conjugacy class $(g)^{ \pm}$of $g \in G$ denotes the union of the conjugacy classes of $g$ and $g^{-1}$, and $r_{G}$ denotes the number of real conjugacy classes of elements not of prime power order. If $\operatorname{Sm}(G)_{\mathcal{P}(G)} \neq\{0\}$ then $r_{G}>1$, since $\operatorname{Sm}(G)_{\mathcal{P}(G)}$ is a subset of the free abelian group $\operatorname{RO}(G)_{\mathcal{P}(G)}^{\{G\}}$ with rank $r_{G}-1$. Laitinen and Pawałowski [20, Theorem A] showed that for a perfect group $G, \operatorname{Sm}(G)_{\mathcal{P}(G)} \neq\{0\}$ if and only if $r_{G}>1$. Let $\mathrm{P} \Sigma \mathrm{L}_{2}(27)$ be the extension of $\mathrm{PSL}_{2}(27)$ by the group $\operatorname{Aut}\left(\mathbb{F}_{27}\right)$ of automorphisms of the field $\mathbb{F}_{27}$ of 27 elements. Pawałowski and Solomon [33, Theorem B3] showed that for any nonsolvable gap group $G$ with $r_{G}>1$ and $G \not \mathrm{P} \Sigma \mathrm{L}_{2}(27)$, there exist nonisomorphic $\mathcal{P}(G)$-connectively Smith equivalent, $\left\{G^{\text {nil }}\right\}$-free $G$-modules. Here a $G$-module $U$ is called $\left\{G^{\text {nil }}\right\}$-free if $U^{G^{\text {nil }}}=0$. Subsequently, Pawałowski and the author [35] showed that for any nonsolvable group $G$ with $r_{G}>1$ and $G \not \mathrm{P} \Sigma \mathrm{L}_{2}(27)$, Aut $\left(A_{6}\right)$, there exist nonisomorphic $\mathcal{P}(G)$-connectively Smith equivalent, $\left\{G^{\text {nil }}\right\}$-free $G$-modules, by further investigating group theoretic arguments in [33] and by employing geometric arguments in [27]. The automorphism group $\operatorname{Aut}\left(A_{6}\right)$ of the alternating group $A_{6}$ satisfies $r_{\operatorname{Aut}\left(A_{6}\right)}=2$ and is not a gap group. Morimoto [26] showed that $\operatorname{Sm}(G) \subset \operatorname{RO}(G)^{\{\cap 2(G)\}}$ and that any Smith equivalent Aut $\left(A_{6}\right)$-modules are isomorphic: $\operatorname{Sm}\left(\operatorname{Aut}\left(A_{6}\right)\right)=\{0\}$. The group is the firstly found example of an Oliver group $G$ such that $r_{G} \geq 2$ but $\operatorname{Sm}(G)=\{0\}$. We also found examples of solvable Oliver groups satisfying the same conditions, see [34, Proposition 5.3-5.6]. Furthermore, Morimoto [26, Theorem 1.1] showed that there exist nonisomorphic $\mathcal{P}\left(\mathrm{P} \Sigma \mathrm{L}_{2}(27)\right)$-connectively Smith equivalent $\mathrm{P} \Sigma \mathrm{L}_{2}(27)$-modules. One of these $\mathrm{P} \Sigma \mathrm{L}_{2}(27)$-modules is $\left\{G^{\text {nil }}\right\}$-free but another is not. Such Smith equivalent modules are also found in $[28,29]$. We studied $\operatorname{PSm}^{c}(G)$ for nonsolvable groups $G$ such that $G / G^{\text {nil }}$ is a cyclic group of order 1, 2, or 3 in [51]. 
We have the implementation that

$$
\operatorname{PSm}^{c}(G) \subset \operatorname{Sm}(G)_{\mathcal{P}(G)} \subset \operatorname{RO}(G)^{\{\cap 2(G)\}} \cap \operatorname{RO}(G)_{\mathcal{P}(G)} .
$$

In this paper we will show that there are many nonsolvable groups $G$ satisfying the equality

$$
\operatorname{PSm}^{c}(G)=\operatorname{RO}(G)^{\{\cap 2(G)\}} \cap \operatorname{RO}(G) \mathcal{P}(G) .
$$

For this purpose, we introduce a sufficient condition for a gap Oliver group satisfying the above equality.

DEFINITION 0.1. A pair $(U, V)$ of real $G$-modules is called $\mathcal{P}(G)$-matched if $[U]-$ $[V] \in \operatorname{RO}(G)_{\mathcal{P}(G)}$. For a family $\mathcal{L}$ of subgroups of $G$, a real $G$-module $W$ is called $\mathcal{L}$-free if $W^{L}=0$ for any $L \in \mathcal{L}$, and a pair $(U, V)$ of real $G$-modules is called a $\mathcal{P}(G)$-matched pair of type $1_{\mathcal{L}}$ if $U^{L}=U^{G} \cong \mathbb{R}, V^{L}=V^{G}=0$ for any $L \in \mathcal{L}$ and $[U]-[V] \in \operatorname{RO}(G)_{\mathcal{P}(G)}$. We say that $G$ satisfies the Nil-P-condition if there are real $G$-modules of type $1_{\left\{G^{\text {nil }}\right\}}$. Let $\mathcal{L}(G)$ be the set of all subgroups $H$ of $G$ such that $H$ includes the smallest normal subgroup of $G$ with p-power index for some prime $p$ and let $\mathcal{M}_{\mathbb{R}}$ (resp. $\mathcal{L}_{\mathbb{R}}$ ) denote the family of all finite groups $G$ for which there exist $\mathcal{P}(G)$-matched pairs of real $G$-modules of type $1_{\{G\}}$ (resp. $\left.1_{\mathcal{L}(G)}\right)$.

Clearly $\mathcal{L}_{\mathbb{R}} \subset \mathcal{M}_{\mathbb{R}}$ and if $G$ satisfies the Nil-P-condition then $G \in \mathcal{L}_{\mathbb{R}}$. If a Sylow 2subgroup of $G$ is a normal subgroup of $G$ then $G \notin \mathcal{M}_{\mathbb{R}}$ and in particular $G$ does not satisfy the Nil-P-condition.

THEOREM 0.2. Let $G$ be a finite group possessing a quotient group which is a gap Oliver group satisfying the Nil-P-condition. Then

$$
\operatorname{PSm}^{c}(G)=\operatorname{RO}(G)^{\{\cap 2(G)\}} \cap \operatorname{RO}(G) \mathcal{P}(G) .
$$

Let $\mathcal{G}$ be the family of finite simple groups $\mathrm{SL}_{2}(4) \cong \mathrm{PSL}_{2}(5) \cong A_{5}, \mathrm{PSL}_{2}(7) \cong$ $\mathrm{PSL}_{3}(2), \mathrm{SL}_{2}(8), \mathrm{PSL}_{2}(9) \cong A_{6}, \mathrm{PSL}_{2}(17), \mathrm{PSL}_{3}(4), \mathrm{SL}_{3}(8), \mathrm{SU}_{3}(3), \mathrm{SU}_{3}(4), \mathrm{PSU}_{3}(8)$, $\mathrm{Sz}(8)$, and $\mathrm{Sz}$ (32). For a positive integer $n, C_{n}$ denotes a cyclic group of order $n$ and $D_{2 n}$ denotes a dihedral group of order $2 n$.

THEOREM 0.3. Let $n$ be an integer and $q$ a prime power $>1$. If a finite group $G$ has a quotient group isomorphic to some nonsolvable group (1)-(6) listed below, then

$$
\operatorname{PSm}^{c}(G)=\operatorname{RO}(G)^{\{\cap 2(G)\}} \cap \operatorname{RO}(G) \mathcal{P}(G) .
$$

(1) A perfect group containing a nonabelian simple group $H$ with $H \notin \mathcal{G}$.

(2) A perfect group containing a subgroup isomorphic to $L \times C_{2}$ with $L \in \mathcal{G}$.

(3) A symmetric group $S_{n}$ with $n \geq 7$.

(4) A projective general linear group $\mathrm{PGL}_{2}(q)$ with $q \neq 2,3,4,5,7,8,9,17$.

(5) A projective general linear group $\mathrm{PGL}_{3}(q)$ with $q \neq 2,4,8$.

(6) A projective general linear group $\mathrm{PGL}_{n}(q)$ with $n \geq 4$.

Acknowledgement. The author would like to thank the anonymous referee(s) for his/their careful reading of this manuscript and the incisive comments. 
1. Nil- $\mathcal{P}$-condition and the $\mathcal{P}(G)$-connective Smith set. For a finite group $G, \mathcal{P}(G)$ denotes the set of all subgroups of $G$ of prime power order, possibly 1 . Let $\mathcal{N}_{2}(G):=\{H \triangleleft G \mid$ $[G: H] \leq 2\}$ and $\cap 2(G):=\cap_{H \in \mathcal{N}_{2}(G)} H$. Then $G / \cap 2(G)$ is a (maximal) elementary abelian 2-group. We put

$$
\begin{aligned}
\operatorname{RO}(G)_{\mathcal{P}(G)} & =\bigcap_{P \in \mathcal{P}(G)} \operatorname{ker}\left(\operatorname{Res}_{P}^{G}: \operatorname{RO}(G) \rightarrow \operatorname{RO}(P)\right), \\
\operatorname{RO}(G)^{\mathcal{N}} & =\bigcap_{N \in \mathcal{N}} \operatorname{ker}\left(\operatorname{Fix}^{N}: \operatorname{RO}(G) \rightarrow \operatorname{RO}(G / N)\right),
\end{aligned}
$$

and

for a family $\mathcal{N}$ of normal subgroups of $G$.

$$
\operatorname{RO}(G)_{\mathcal{P}(G)}^{\mathcal{N}}=\operatorname{RO}(G)_{\mathcal{P}(G)} \cap \operatorname{RO}(G)^{\mathcal{N}},
$$

A finite group $G$ is called a gap group (cf. [30, 46, 47, 49, 50, 52]) if there exists a real $G$-module $W$ such that

(1) $W$ is $\mathcal{L}(G)$-free and

(2) $\operatorname{dim} W^{P}>2 \operatorname{dim} W^{H}$ for any subgroups $P, H$ of $G$ with $P \in \mathcal{P}(G)$ and $P<H$.

Recall the following theorem.

THEOREM 1.1 ([26, Lemma 4.6]). Let $G$ be a gap Oliver group. Let $\left(U_{1}, U_{2}\right)$ and $\left(U_{3}, U_{4}\right)$ be $\mathcal{P}(G)$-matched pairs of $G$-modules of type $1_{\mathcal{N}_{2}(G)}$ such that $U_{2}^{G^{\mathrm{nil}}}=0=U_{4}^{G^{\mathrm{nil}}}$. If $\left(U_{1}, U_{3}\right)$ is a $\mathcal{P}(G)$-matched pair, then $\left[U_{1}\right]-\left[U_{3}\right] \in \operatorname{PSm}^{c}(G)$.

We obtain the following lemma by this theorem.

LEMMA 1.2. Let $G$ be a gap Oliver group. Suppose that for any $\eta \in \operatorname{RO}\left(G / G^{\mathrm{nil}}\right)$ there is an element $X_{\eta} \in \operatorname{RO}(G)^{\left\{G^{\mathrm{nil}}\right\}}$ such that $\eta+X_{\eta} \in \operatorname{RO}(G)_{\mathcal{P}(G)}$. Then $\operatorname{PSm}^{c}(G)=$ $\operatorname{RO}(G)_{\mathcal{P}(G)}^{\mathcal{N}_{2}(G)}$.

Proof. Since $\operatorname{PSm}^{c}(G) \subset \operatorname{RO}(G)_{\mathcal{P}(G)}^{\mathcal{N}_{2}(G)}$, we show that any element of $\operatorname{RO}(G)_{\mathcal{P}(G)}^{\mathcal{N}_{2}(G)}$ lies in $\operatorname{PSm}^{c}(G)$. Let $\left(A_{1}, A_{2}\right)$ be a pair of $\left\{G^{\text {nil }}\right\}$-free real $G$-modules such that $\mathbb{R} \oplus A_{1}$ and $A_{2}$ are $\mathcal{P}(G)$-matched. Let $X \in \operatorname{RO}(G)_{\mathcal{P}(G)}^{\mathcal{N}_{2}(G)}$. Write $X$ as $\left[X_{1}\right]-\left[X_{2}\right]$ by using $\mathcal{N}_{2}(G)$-free real $G$-modules $X_{1}$ and $X_{2}$. By the assumption, for $j=1,2$, there exist $\left\{G^{\text {nil }}\right\}$-free real $G$-modules $Y_{j+}$ and $Y_{j-}$ such that $\left(X_{j}^{G^{\text {nil }}} \oplus Y_{j+}, Y_{j-}\right)$ is an $\mathcal{N}_{2}(G)$-free $\mathcal{P}(G)$-matched pair. Since $X_{j}=\left(X_{j}-X_{j}^{G^{\mathrm{nil}}}\right) \oplus X_{j}^{G^{\mathrm{nil}}}$, we see that

$$
\left(\mathbb{R} \oplus A_{1} \oplus X_{j} \oplus Y_{j+}, A_{2} \oplus\left(X_{j}-X_{j}^{G^{\mathrm{nil}}}\right) \oplus Y_{j-}\right)
$$

is a $\mathcal{P}(G)$-matched pair of type $1_{\{G\}}$. Put

$$
\begin{aligned}
& U_{1}=\mathbb{R} \oplus X_{1} \oplus A_{1} \oplus Y_{1+} \oplus Y_{2+}, \\
& U_{2}=\left(X_{1}-X_{1}^{G^{\mathrm{nil}}}\right) \oplus A_{2} \oplus Y_{1-} \oplus Y_{2+}, \\
& U_{3}=\mathbb{R} \oplus X_{2} \oplus A_{1} \oplus Y_{1+} \oplus Y_{2+}, \quad \text { and } \\
& U_{4}=\left(X_{2}-X_{2}^{G^{\mathrm{nil}}}\right) \oplus A_{2} \oplus Y_{1+} \oplus Y_{2-} .
\end{aligned}
$$


Then we see that $U_{1}^{\cap 2(G)}=\mathbb{R}, U_{3}^{\cap 2(G)}=\mathbb{R}, U_{2}^{G^{\mathrm{nil}}}=0=U_{4}^{G^{\mathrm{nil}}}$, and $\left(U_{1}, U_{2}\right),\left(U_{3}, U_{4}\right)$ and $\left(U_{1}, U_{3}\right)$ are all $\mathcal{P}(G)$-matched pairs. Therefore, we have $X=\left[U_{1}\right]-\left[U_{3}\right] \in \operatorname{PSm}^{c}(G)$ by Theorem 1.1.

The following lemma connects with the Nil-P-condition.

LEMMA 1.3. If a group $G$ satisfies the Nil-P-condition then for any $\eta \in \operatorname{RO}\left(G / G^{\mathrm{nil}}\right)$, there exists an element $X \in \operatorname{RO}(G)^{\left\{G^{\mathrm{nil}}\right\}}$ such that $\eta+X \in \operatorname{RO}(G)_{\mathcal{P}(G)}$.

Proof. Since $G$ satisfies the Nil-P-condition, there is $X \in \operatorname{RO}(G)^{\left\{G^{\text {nil }}\right\}}$ such that $[\mathbb{R}]+$ $X \in \operatorname{RO}(G)_{\mathcal{P}(G)}$. Put $Y=\eta \cdot([\mathbb{R}]+X)=\eta+\eta \cdot X$. It is easy to see that $\eta \cdot X \in \operatorname{RO}(G)^{\left\{G^{\mathrm{nil}}\right\}}$ and $Y \in \operatorname{RO}(G)_{\mathcal{P}(G)}$.

Putting Lemmas 1.2 and 1.3 together, we immediately see the following theorem.

THEOREM 1.4. For a gap Oliver group $G$ satisfying the Nil-P-condition, it holds that

$$
\operatorname{PSm}^{c}(G)=\operatorname{Sm}(G)_{\mathcal{P}(G)}=\operatorname{RO}(G)_{\mathcal{P}(G)}^{\mathcal{N}_{2}(G)} .
$$

In particular, $\operatorname{PSm}^{c}(G)$ is an additive group.

Two elements $x$ and $y$ of $G$ are called real conjugate if $x$ is conjugate to $y$ or $y^{-1}$, and $(x)^{ \pm}$denotes the real conjugacy class of $x$. The set $\operatorname{RO}(G)_{\mathcal{P}(G)}$ is a free abelian group with rank $r_{G}$, where $r_{G}$ is the cardinality of the set $\overline{\mathrm{NPP}}(G)$ consisting of real conjugacy classes of elements of $G$ not of prime power order.

COROLlary 1.5. For a gap Oliver group $G$ satisfying the Nil-P-condition, if there are two elements $x$ and $y$ of $G$ not of prime power order such that they are not real conjugate and $f(x)$ and $f(y)$ are conjugate where $f$ is a natural surjective homomorphism from $G$ to $G / \cap 2(G)$, then $\operatorname{PSm}^{c}(G) \neq 0$.

Proof. By the assumption, the Second Rank Lemma [33, p. 856] yields

$$
\operatorname{rank} \operatorname{RO}(G)_{\mathcal{P}(G)}^{\mathcal{N}_{2}(G)}=r_{G}-r_{G, \cap 2(G)}>0
$$

since $\operatorname{RO}(G)_{\mathcal{P}(G)}^{\mathcal{N}_{2}(G)}=\operatorname{RO}(G)_{\mathcal{P}(G)}^{\{\cap 2(G)\}}$, where $r_{G, \cap 2(G)}$ is the cardinality of the set $\left\{(f(a))^{ \pm}\right.$। $\left.(a)^{ \pm} \in \overline{\operatorname{NPP}}(G)\right\}$. Therefore the assertion follows from Theorem 1.4.

2. Induced modules and the Nil- $\mathcal{P}$-condition. If $G$ satisfies the Nil- $\mathcal{P}$-condition, then $G$ and $G^{\text {nil }}$ lie in $\mathcal{M}_{\mathbb{R}}$ by definition. A perfect group $G$ satisfies the Nil- $\mathcal{P}$-condition if and only if $G \in \mathcal{M}_{\mathbb{R}}$.

THEOREM 2.1. If there are a subgroup $K$ of $G$ and a surjective homomorphism $f: K \rightarrow H$ such that $f\left(K \cap G^{\text {nil }}\right)=H, K G^{\text {nil }}=G$ and $H \in \mathcal{M}_{\mathbb{R}}$, then $G$ satisfies the Nil-P-condition.

Proof. Let $\left(W_{1}, W_{2}\right)$ be a $\mathcal{P}(H)$-matched pair of real $H$-modules of type $1_{\{H\}}$. Put $U=\operatorname{Ind}_{K}^{G} f^{*}\left(W_{1}\right)$ and $V=\operatorname{Ind}_{K}^{G} f^{*}\left(W_{2}\right)$. We show that they are required real $G$-modules. 
Clearly $U$ and $V$ are $\mathcal{P}(G)$-matched and $\operatorname{dim} U^{G}=\operatorname{dim}\left(f^{*}\left(W_{1}\right)\right)^{K}=\operatorname{dim} W_{1}^{H}=1$. Furthermore we have $\operatorname{dim} U^{G^{\text {nil }}}=1$ and $\operatorname{dim} V^{G^{\text {nil }}}=0$, since

$$
\begin{aligned}
\operatorname{dim}\left(\operatorname{Ind}_{K}^{G} f^{*}(W)\right)^{G^{\mathrm{nil}}} & =\sum_{G^{\mathrm{nil}} g K \in G^{\mathrm{nil}} \backslash G / K} \operatorname{dim}\left(f^{*}(W)\right)^{K \cap g^{-1} G^{\mathrm{nil}} g} \\
& =\operatorname{dim}\left(f^{*}(W)\right)^{K \cap G^{\mathrm{nil}}} \\
& =\operatorname{dim} W^{H}
\end{aligned}
$$

for a real $H$-module $W$. Therefore $G$ satisfies the Nil- $\mathcal{P}$-condition.

Proposition 2.2. Let $f: G \rightarrow K$ be a surjective homomorphism. Let $U$ and $V$ be $\left\{K^{\text {nil }}\right\}$-free real $K$-modules. If $\mathbb{R} \oplus U$ and $V$ are $\mathcal{P}(K)$-matched, then $f^{*} U$ and $f^{*} V$ are $\left\{G^{\text {nil }}\right\}$-free real $G$-modules such that $\mathbb{R} \oplus f^{*} U$ and $f^{*} V$ are $\mathcal{P}(G)$-matched. In particular, if $K$ satisfies the Nil-P-condition then $G$ satisfies the Nil-P-condition.

Proof. The assertion follows from Theorem 2.1 because $f\left(G^{\text {nil }}\right)=K^{\text {nil }}$.

COROLlary 2.3. Let $G$ and $K$ be finite groups. Let $U$ and $V$ be $\left\{G^{\mathrm{nil}}\right\}$-free real $G$ modules. If $[\mathbb{R} \oplus U]-[V] \in \operatorname{RO}(G)_{\mathcal{P}(G)}$, then $\left(f^{*}(\mathbb{R} \oplus U), f^{*}(V)\right)$ is a $\mathcal{P}(G \times K)$-matched pair of type $1_{G^{\mathrm{nil}}}$, where $f: G \times K \rightarrow G$ is a canonical surjective homomorphism.

Proposition 2.4. Let $G$ be a finite group satisfying the Nil-P-condition and $K a$ subgroup with $K \geq G^{\text {nil }}$. If $G^{\text {nil }}$ is a perfect group then $K$ satisfies the Nil-P-condition.

Proof. Let $U$ and $V$ be real $G$-modules such that $U^{G^{\text {nil }}}=V^{G^{\text {nil }}}=0$ and $[\mathbb{R} \oplus U]-$ $[V] \in \operatorname{RO}(G)_{\mathcal{P}(G)}$. Since $G^{\text {nil }}=K^{\text {nil }}$, real $K$-modules $\operatorname{Res}_{K}^{G} U$ and $\operatorname{Res}_{K}^{G} V$ satisfy the required condition for the Nil- $\mathcal{P}$-condition.

3. Groups satisfying the Nil- $\mathcal{P}$-condition. In this section we show a family of nonsolvable groups satisfying the Nil- $\mathcal{P}$-condition. Note that if $G$ satisfies the Nil- $\mathcal{P}$-condition then $G^{\text {nil }} \in \mathcal{M}_{\mathbb{R}}$ and recall that $G \in \mathcal{M}_{\mathbb{R}}$ if and only if $G$ has a subquotient group isomorphic to a dihedral group $D_{2 p q}$ of order $2 p q$, where $p$ and $q$ are distinct primes [31, Lemma 3.1 (b)].

Proposition 3.1. If $K \notin \mathcal{M}_{\mathbb{R}}$ then $K \times P \notin \mathcal{M}_{\mathbb{R}}$ for a group $P$ of odd order.

Proof. Suppose that $K \times P \in \mathcal{M}_{\mathbb{R}}$ and then there are a subgroup $H$ of $K \times P$, distinct primes $p, q$, and a surjective homomorphism $f: H \rightarrow D_{2 p q}$. Let $D_{2 p q}=\langle a, b| a^{2}=b^{p q}=$ $\left.(a b)^{2}=e\right\rangle$. Take a 2-element $x$ of $f^{-1}(a)$. Then $x \in K$ since $|P|$ is odd. For $y \in f^{-1}(\langle b\rangle)$, we see that $f\left(x^{-1} y x\right)=f(y)^{-1}$. In particular if the order of $f(y)$ is an odd integer greater than 1 , then $x^{-1} y x \neq y$. Since $x$ commutes any element of $P, P \cap H$ is a subgroup of $\operatorname{Ker} f$. Hence, there is a surjective homomorphism $H P / P \cong H /(P \cap H) \rightarrow H / \operatorname{Ker} f \cong D_{2 p q}$. Since $K \cong K P / P, K$ has a subquotient group isomorphic to $D_{2 p q}$.

If $P$ is of even order and $K$ has a subquotient group isomorphic to a dihedral group $D_{2 p}$ for an odd prime $p$, then $K \times P \in \mathcal{M}_{\mathbb{R}}$, since $D_{4 p} \cong D_{2 p} \times C_{2}$. 
We consider perfect groups. For a nontrivial perfect group $K$, it holds that $\operatorname{PSm}^{c}(K)=$ $\operatorname{RO}(K)_{\mathcal{P}(K)}^{\{K\}}$ [20], however it is unknown whether $\operatorname{PSm}^{c}(G)=\operatorname{RO}(G)_{\mathcal{P}(G)}^{\mathcal{N}_{2}(G)}$ even for $G=$ $K \times C_{6} \times C_{6}$.

Proposition 3.2. $K \times C_{2} \in \mathcal{M}_{\mathbb{R}}$ for any $K \in \mathcal{G}$.

Proof. Let $K \in \mathcal{G}$. For conjugacy classes $c_{1}, c_{2}, c_{3}$ in $K$ and $z \in c_{3}$, the class algebra constant of $c_{1}, c_{2}, c_{3}$ is the number of pairs $(x, y)$ with $x \in c_{1}, y \in c_{2}$ and $x y=z$. We take $c_{1}, c_{2}$ and $c_{3}$ as conjugacy classes of elements of order $2, r, 2$, respectively, where $r$ is an integer. If the class algebra constant of $c_{1}, c_{2}, c_{3}$ is positive, then $K$ has a dihedral subgroup of order $2 r$. Class algebra constants are computable by the character table. By using [8], we see that if $K$ is a Suzuki group then $K$ has a dihedral subgroup of order 10 and otherwise $K$ has a dihedral subgroup of order 6 . Therefore $K \times C_{2}$ has a dihedral subgroup of order 20 or 12 .

THEOREM 3.3. A nonabelian simple group $G$ satisfies the Nil-P-condition if and only if $G \notin \mathcal{G}$.

PROOF. For a perfect group $G, G$ satisfies the Nil-P-condition if and only if $G \in \mathcal{M}_{\mathbb{R}}$. It suffices to show that $G$ contains $D_{2 p q}$ for some distinct primes $p, q$. Every nonabelian simple group is alternating, or in one of 16 families of groups of Lie type, or one of 26 sporadic groups. We check the assertion for each of these families.

Since the subgroup $\langle(1,2)(5,6),(1,2)(3,4)(5,6,7)\rangle$ is a dihedral group $D_{12}$ of order 12 , the alternating groups $A_{n}$ for $n \geq 7$ lies in $\mathcal{M}_{\mathbb{R}}$.

Let $G$ be a nonabelian simple group of Lie type. We use the notation as (twisted) Chevalley groups (see [8, xiv-xvi]). First, we consider the case when $G=\mathrm{A}_{n}(q) \cong \operatorname{PSL}_{n+1}(q)$. The maximal dihedral subgroups of $\mathrm{A}_{1}(q)$ are $D_{2(q \pm 1) / d}$, where $d=\operatorname{gcd}(n, q-1)$ (cf. [16, Theorem 6.5.1]). Then $\mathrm{A}_{1}(q) \notin \mathcal{M}_{\mathbb{R}}$ implies $q=2,3,4,5,7,9,17$. We directly see that $\mathrm{A}_{2}(q)$ for odd $q$ and $\mathrm{A}_{3}(2)$ contain $D_{12}$. Note that $\mathrm{A}_{3}\left(2^{a}\right), a \geq 2$ contains a subgroup $\mathrm{A}_{3}(2)$. Therefore $G \in \mathcal{M}_{\mathbb{R}}$ if $G \notin \mathcal{G}$.

Next we consider projective special unitary groups $G={ }^{2} \mathrm{~A}_{n}\left(q, q^{2}\right)={ }^{2} \mathrm{~A}_{n}(q)$ for $n \geq 2$. Since $G$ contains ${ }^{2} \mathrm{~A}_{1}(q) \cong \mathrm{A}_{1}(q), G \in \mathcal{M}_{\mathbb{R}}$ if $q \neq 2,3,4,5,7,8,9,17$. For $q=5,7,17$, ${ }^{2} \mathrm{~A}_{2}(q)$ contains $D_{12}$ and ${ }^{2} \mathrm{~A}_{2}(9)$ contains $D_{20}$ by using the character table [42, Table 2]. Since ${ }^{2} \mathrm{~A}_{3}(2)$ contains $D_{12},{ }^{2} \mathrm{~A}_{3}(q)$ for $q=2,4,8$ lies in $\mathcal{M}_{\mathbb{R}}$. Therefore if $G \notin \mathcal{G}$ then $G \in \mathcal{M}_{\mathbb{R}}$.

Now we consider orthogonal groups $\mathrm{D}_{m}(q)$ and ${ }^{2} \mathrm{D}_{m}(q)$ for $m \geq 4, \mathrm{~B}_{n}(q)$ for $n \geq 2$, and projective symplectic groups $\mathrm{C}_{k}(q)$ for $k \geq 3$. $\mathrm{D}_{3}(q) \cong \mathrm{A}_{3}(q)$ implies that $\mathrm{D}_{m}(q) \in \mathcal{M}_{\mathbb{R}}$ and ${ }^{2} \mathrm{D}_{3}(q) \cong{ }^{2} \mathrm{~A}_{3}(q)$ implies that ${ }^{2} \mathrm{D}_{m}(q) \in \mathcal{M}_{\mathbb{R}}$. Since $\mathrm{B}_{2}(q)$ contains ${ }^{2} \mathrm{D}_{2}(q) \cong \mathrm{A}_{1}\left(q^{2}\right)$, if $q \neq 2,3$ then ${ }^{2} \mathrm{~B}_{2}(q) \in \mathcal{M}_{\mathbb{R}}$. $\mathrm{B}_{2}(2)$ is isomorphic to $S_{6}$ and $\mathrm{B}_{2}(3)$ contains $D_{12}$ and $S_{6}$ by [15]. Thus $\mathrm{B}_{2}(q) \in \mathcal{M}_{\mathbb{R}}$ for $q=2,3$, and consequently $\mathrm{B}_{2}(q) \in \mathcal{M}_{\mathbb{R}}$ for any $q$. Since $\mathrm{C}_{2}(q) \cong \mathrm{B}_{2}(q), \mathrm{C}_{k}(q) \in \mathcal{M}_{\mathbb{R}}$ holds. 
Simple groups $\mathrm{E}_{6}(q), \mathrm{E}_{7}(q), \mathrm{E}_{8}(q), \mathrm{F}_{4}(q),{ }^{2} \mathrm{E}_{6}(q)$ contains $\mathrm{D}_{5}(q), \mathrm{D}_{6}(q), \mathrm{D}_{8}(q), \mathrm{B}_{4}(q)$, ${ }^{2} \mathrm{D}_{5}(q)$, respectively, by [21, Table 1$]$, which lie in $\mathcal{M}_{\mathbb{R}}$. $\mathrm{G}_{2}(4)$ contains $\mathrm{A}_{1}(13)$ by [9, Theorem (2.2)] and thus $\mathrm{G}_{2}\left(2^{a}\right)$ for $a \geq 2$ lies in $\mathcal{M}_{\mathbb{R}}$ (cf. [9, Theorem (2.3)]). Since $\mathrm{G}_{2}(q)$ for odd $q \geq 4$ contains $\mathrm{A}_{2}(q)$ by [17, Theorem A], it lies in $\mathcal{M}_{\mathbb{R}}$. Noting that $\mathrm{G}_{2}(2)$ and $\mathrm{G}_{2}(3)$ are not simple, the simple group $\mathrm{G}_{2}(q)$ lies in $\mathcal{M}_{\mathbb{R}}$. The Ree group ${ }^{2} \mathrm{G}_{2}\left(3^{2 n+1}\right)$ for $n \geq 1$ contains $\mathrm{A}_{1}\left(3^{2 n+1}\right) \in \mathcal{M}_{\mathbb{R}}$ by $\left[17\right.$, Theorem C]. Therefore ${ }^{2} \mathrm{G}_{2}\left(3^{2 n+1}\right) \in \mathcal{M}_{\mathbb{R}}$ for any $n \geq 1$.

The Steinberg group ${ }^{3} \mathrm{D}_{4}(q)$ contains $\mathrm{A}_{1}(q) \times \mathrm{A}_{1}\left(q^{3}\right)$ if $q=2^{a}$ and $\operatorname{SL}_{2}\left(q^{3}\right)$ if $q$ is odd [18]. Therefore ${ }^{3} \mathrm{D}_{4}(q) \in \mathcal{M}_{\mathbb{R}}$ for any $q$. Similarly, ${ }^{2} \mathrm{~F}_{4}\left(2^{2 n+1}\right)$ contains ${ }^{2} \mathrm{~A}_{2}\left(2^{2 n+1}\right)$ for $n \geq 1$ [22] and then lies in $\mathcal{M}_{\mathbb{R}}$. The Tits group ${ }^{2} \mathrm{~F}_{4}(2)^{\prime}$ contains $\mathrm{A}_{1}(25)$ [55, 54, Theorem $1]$, and then ${ }^{2} \mathrm{~F}_{4}(2)^{\prime}$ lies in $\mathcal{M}_{\mathbb{R}}$. For the Suzuki group ${ }^{2} \mathrm{~B}_{2}\left(2^{2 n+1}\right) \cong \mathrm{S} z\left(2^{2 n+1}\right)$, there is an element $x$ of $\mathrm{S} z\left(2^{2 n+1}\right)$ of order $2^{2 n+1}-1$ such that the normalizer of $x$ is a dihedral group of order $2\left(2^{2 n+1}-1\right)$ [53, Theorem 9]. More precisely, by the character table [4, Table 8], $\mathrm{S} z\left(2^{2 n+1}\right)$ contains dihedral groups $D_{2\left(2^{2 n+1} \pm 2^{n+1}+1\right)}$. Thus $\mathrm{S} z\left(2^{2 n+1}\right) \in \mathcal{M}_{\mathbb{R}}$ for $n \geq 3$.

For sporadic groups $G$, by the character table of $G$ [8], we compute the class algebra constants and show that $G$ contains $D_{12}$. Hence $G \in \mathcal{M}_{\mathbb{R}}$.

Therefore we conclude that every nonabelian simple group $\notin \mathcal{G}$ lies in $\mathcal{M}_{\mathbb{R}}$.

Finally let $G \in \mathcal{G}$. If $G \neq \mathrm{SU}_{3}(3), \mathrm{SU}_{3}(4), \mathrm{SL}_{3}(8), \mathrm{PSU}_{3}(8)$, every element is of prime power order and thus $G$ has no cyclic subquotient group of order $p q$ for any distinct primes $p, q$, which implies $G \notin \mathcal{M}_{\mathbb{R}}$. For $G=\mathrm{SU}_{3}(3)$, in GAP [15], we get the group $G$ by 'SU(3,3)' and all subgroups of $G$ up to conjugacy by using the function 'ConjugacyClassesSubgroups'. Since $G$ is simple, $G$ has no quotient dihedral group. If a proper subgroup $H$ of $G$ has order divisible by $2 p q$ for distinct primes $p, q$, then $p q=15$ and $H$ has no quotient dihedral group of order $2 p q$. Therefore there is no dihedral subquotient group $D_{2 p q}$ of $G$ for any distinct primes $p, q$, which implies $G \notin \mathcal{M}_{\mathbb{R}}$. For $G=\mathrm{SU}_{3}(4), \mathrm{SL}_{3}(8), \mathrm{PSU}_{3}(8)$, similarly we get $G \notin \mathcal{M}_{\mathbb{R}}$.

Next we consider symmetric groups and (projective) general linear groups. For a perfect group $G, G \in \mathcal{M}_{\mathbb{R}}$ implies $G \in \mathcal{L}_{\mathbb{R}}$. If $\left[G: G^{\text {nil }}\right]$ is a prime power, then $G$ satisfies the Nil- $\mathcal{P}$-condition if and only if $G \in \mathcal{L}_{\mathbb{R}}$.

Proposition 3.4 ([48, Proposition 2.14]). For $n \geq 7$, the symmetric group $S_{n}$ satisfy the Nil-P-condition.

ProOF. Let $K$ be a subgroup of $S_{n}$ generated by three elements $(1,2)(4,5),(1,2,3)$ $(4,5)$ and $(6,7)$. Then $K$ is isomorphic to $D_{12} \times C_{2}$. A canonical surjective homomorphism $f: K \rightarrow D_{12}$ satisfies the condition of Theorem 2.1 .

Note that $A_{5} \times C_{2} \in \mathcal{M}_{\mathbb{R}}$, and $A_{5} \times C_{2}$ does not satisfy the Nil-P-condition while $\operatorname{PSm}^{c}\left(A_{5} \times C_{2}\right)=\operatorname{RO}\left(A_{5} \times C_{2}\right)_{\mathcal{P}\left(A_{5} \times C_{2}\right)}^{\left\{A_{5}\right\}}$ holds. We consider projective general linear groups $\operatorname{PGL}_{n}(q)$. For $n=2,3,4$, since $\left[\operatorname{PGL}_{n}(q): \operatorname{PSL}_{n}(q)\right]=\operatorname{gcd}(n, q-1)$, the index $\left[\operatorname{PGL}_{n}(q)\right.$ : $\left.\operatorname{PGL}_{n}(q)^{\text {nil }}\right]$ is $1,2,3$, or $2^{2}$.

LEMMA 3.5. If $q \neq 2,3,4,5,7,8,9,17$, then the projective general linear group $\mathrm{PGL}_{2}(q)$ satisfies the Nil-P-condition. 
PROOF. If $q$ is a power of 2 , since $\operatorname{PGL}_{2}(q) \cong \operatorname{PSL}_{2}(q)$ the assertion follows from Theorem 3.3. Suppose that $q$ is odd. Since $q \neq 3,5,7,9,17$, there exist integers $\varepsilon, a, b$ such that $\varepsilon= \pm 1, q+\varepsilon=a b, a$ is even, $a \geq 4, b \geq 2$, and $\operatorname{gcd}(a, b)=1$. Let $x_{q-1}, x_{q}, x_{q+1}$ be elements of $\mathrm{PGL}_{2}(q)$ of order $q-1, q, q+1$ respectively and let $V_{q}, V_{q+1}^{(i)}(1 \leq i<(q-1) / 2)$, and $V_{q-1}^{(j)}(1 \leq j<(q+1) / 2)$ be irreducible real $\operatorname{PGL}_{2}(q)$-modules determined by the following character values:

$$
\begin{aligned}
& \chi_{V_{q}}(e)=1, \quad \chi_{V_{q}}\left(x_{q}\right)=0, \chi_{V_{q}}\left(x_{q-1}^{h}\right)=1, \quad \chi_{V_{q}}\left(x_{q+1}^{k}\right)=-1, \\
& \chi_{V_{q+1}^{(i)}}(e)=q+1, \quad \chi_{V_{q+1}^{(i)}}\left(x_{q}\right)=1, \quad \chi_{V_{q+1}^{(i)}}\left(x_{q-1}^{h}\right)=\eta^{i h(q+1)}+\eta^{-i h(q+1)}, \\
& \chi_{V_{q+1}^{(i)}}\left(x_{q+1}^{k}\right)=0, \quad \chi_{V_{q-1}^{(j)}}(e)=q-1, \quad \chi_{V_{q-1}^{(j)}}\left(x_{q}\right)=-1, \quad \chi_{V_{q-1}^{(j)}}\left(x_{q-1}^{h}\right)=0, \\
& \chi_{V_{q-1}^{(j)}}\left(x_{q+1}^{k}\right)=-\eta^{j k(q-1)}-\eta^{-j k(q-1)},
\end{aligned}
$$

for $1 \leq h \leq(q-1) / 2$ and $1 \leq k \leq(q+1) / 2$, where $e$ denotes the identity element and $\eta=$ $\exp \left(2 \pi \sqrt{-1} /\left(q^{2}-1\right)\right)$ (cf. [44, Table III]). For $s=\min (a, q+\varepsilon-a), t=\min (b, q+\varepsilon-b)$, $u=\min (s+t, q+\varepsilon-s-t)$, we see that $1 \leq s, t, u \leq(q+\varepsilon) / 2$ and that the pair

$$
\left(\mathbb{R}^{\oplus(1+\varepsilon) / 2} \oplus V_{q+\varepsilon}^{(s)} \oplus V_{q+\varepsilon}^{(t)}, \mathbb{R}^{\oplus(1-\varepsilon) / 2} \oplus V_{q} \oplus V_{q+\varepsilon}^{(u)}\right)
$$

of $\operatorname{PGL}_{2}(q)$-modules is a $\mathcal{P}\left(\operatorname{PGL}_{2}(q)\right)$-matched pair of type $1_{\left\{\mathrm{PGL}_{2}(q)^{\text {nill }}\right.}$.

Proposition 3.6 (cf. [48, Lemma 4.1.1]). Let $m>n$ and suppose that $\mathrm{PGL}_{n}(q) \in$ $\mathcal{M}_{\mathbb{R}}$. Then $\mathrm{PGL}_{m}(q)$ satisfies the Nil-P-condition.

Proof. Let $\mathbb{F}_{q}$ be a field consisting of $q$ elements and let $E$ be the $(m-n-1) \times(m-$ $n-1)$ identity matrix. Put

$$
L=\left\{\left(\begin{array}{ccc}
A & O & O \\
O & x \operatorname{det}(A)^{-1} & 0 \\
O & O & E
\end{array}\right) \mid A \in \mathrm{GL}_{n}(q), x \in \mathbb{F}_{q} \backslash\{0\}\right\}
$$

and

$$
L^{\prime}=\left\{\left(\begin{array}{ccc}
A & O & O \\
O & \operatorname{det}(A)^{-1} & O \\
O & O & E_{m-n-1}
\end{array}\right) \mid A \in \mathrm{GL}_{n}(q)\right\} .
$$

The group $L$ is a subgroup of $\mathrm{GL}_{m}(q)$ isomorphic to $\mathrm{GL}_{n}(q) \times C_{q-1}$ and $L^{\prime}$ is a subgroup of $\mathrm{SL}_{m}(q)$ isomorphic to $\mathrm{GL}_{n}(q)$.

Let $g: \operatorname{GL}_{m}(q) \rightarrow \operatorname{PGL}_{m}(q)$ be a natural projection. Put $K=g(L)$ and $H=\operatorname{PGL}_{n}(q)$. The commutative diagram

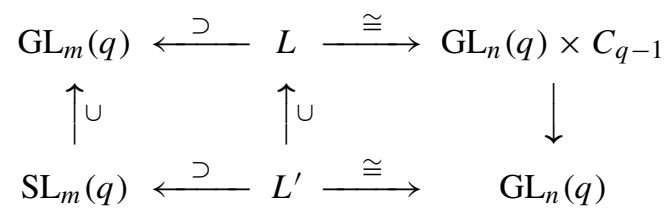


induces the following commutative diagram:

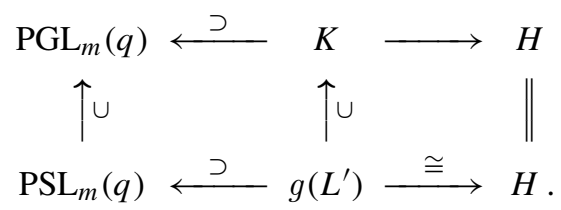

Since $\mathrm{SL}_{m}(q) L=\mathrm{GL}_{m}(q)$ implies $\operatorname{PSL}_{m}(q) K=\operatorname{PGL}_{m}(q)$, the group $\operatorname{PGL}_{m}(q)$ satisfies the Nil-P-condition by Theorem 2.1 .

THEOREM 3.7. Let $q$ be a prime power $>1$. The following groups $G$ satisfy the Nil$\mathcal{P}$-condition.

(1) Projective general linear groups $\operatorname{PGL}_{2}(q), q \neq 2,3,4,5,7,8,9,17$.

(2) Projective general linear groups $\operatorname{PGL}_{3}(q), q \neq 2,4,8$.

(3) Projective general linear groups $\mathrm{PGL}_{n}(q), n \geq 4$.

(4) General linear groups $\mathrm{GL}_{2}(q), q \neq 2,3,4,5,7,8,9,17$.

(5) General linear groups $\mathrm{GL}_{3}(q), q \neq 2,4,8$.

(6) General linear groups $\mathrm{GL}_{n}(q), n \geq 4$.

Proof. The conclusion for the case (1) is implied by Lemma 3.5. Let $n=3$. Note that $\mathcal{L}_{\mathbb{R}}$ is a subfamily of $\mathcal{M}_{\mathbb{R}}$. If $q \neq 2,3,4,5,7,8,9,17$ then $\operatorname{PGL}_{3}(q) \in \mathcal{L}_{\mathbb{R}}$. For $q=3,5,9,17$, since $\operatorname{PGL}_{3}(q)$ is isomorphic to a nonabelian simple group $\operatorname{PSL}_{3}(q)$, we have $\operatorname{PGL}_{3}(q) \in \mathcal{L}_{\mathbb{R}}$ by Theorem 3.3. We see that PGL 3 (7) contains $D_{12}$ by computing class algebra constants [44]. Next, let $n=4$. If $G$ satisfies the Nil-P-condition, then by definition $G$ lie in $\mathcal{M}_{\mathbb{R}}$. If $q \neq 2,4,8$, then $\operatorname{PGL}_{3}(q) \in \mathcal{M}_{\mathbb{R}}$ and thus $\operatorname{PGL}_{4}(q)$ satisfies the Nil- $\mathcal{P}$-condition by Proposition 3.6, and otherwise, $\mathrm{PGL}_{4}(q)$ satisfies the Nil- $\mathcal{P}$-condition by Lemma 3.5, since PGL $4(q)=\mathrm{PSL}_{4}(q)$. Therefore $\mathrm{PGL}_{4}(q)$ satisfies the Nil-P $\mathcal{P}$-condition for $q>1$. Again since $\mathrm{PGL}_{4}(q) \in \mathcal{M}_{\mathbb{R}}, \mathrm{PGL}_{n}(q)$ for $n \geq 5, q>1$ satisfies the Nil-P-condition by Proposition 3.6. The statements for (4), (5), and (6) follow from ones for (1), (2), and (3), respectively by Proposition 2.2 .

4. Proof of Theorems 0.2 and 0.3. In this section we prove Theorems 0.2 and 0.3 .

PROOF OF THEOREM 0.2. If $G$ has a quotient group which is a gap Oliver group satisfying the Nil- $\mathcal{P}$-condition, then $G$ is also a gap Oliver group satisfying the Nil- $\mathcal{P}$-condition by Proposition 2.2 and [46, Theorem 1.2]. Thus Theorem 1.4 implies the assertion.

Proof OF THEOREM 0.3. Let $H$ be an arbitrary group (1)-(6) listed in Theorem 0.3 . Since $H$ is a nonsolvable group, it is an Oliver group. The nontrivial perfect groups [19, 30] and the symmetric groups $S_{n}$ for $n \geq 6[11,30]$, the projective general linear groups $\operatorname{PGL}_{n}(q)$ [47, Corollary 3.5] are gap groups. Thus $H$ is a gap Oliver group. Furthermore, $H$ satisfies the Nil-P-condition by Theorems 3.3, 3.7 and Proposition 3.4.

Since $G$ has a gap Oliver quotient group $H, G$ is also a gap Oliver group by [46, Theorem 1.2], and satisfies the Nil-P $\mathcal{P}$-condition by Proposition 2.2. Therefore, by Theorem 1.4, we have 
the equality

$$
\operatorname{PSm}^{c}(G)=\operatorname{RO}(G)^{\{\cap 2(G)\}} \cap \operatorname{RO}(G) \mathcal{P}(G) .
$$

\section{REFERENCES}

[1] M. F. AтiYAh And R. Bott, A Lefschetz fixed point formula for elliptic differential operators, Bull. Amer. Math. Soc. 72 (1966), 245-250.

[ 2 ] G. E. BREDON, Representations at fixed points of smooth actions of compact groups, Ann. of Math. (2) 89 (1969), 515-532.

[ 3 ] G. E. BREDON, Introduction to compact transformation groups, Pure Appl. Math. 46, Academic Press, New York-London, 1972.

[ 4 ] O. BRUNAT, The Shintani descents of Suzuki groups and their consequences, J. Algebra 303 (2006), 869-890.

[ 5 ] S. E. Cappell And J. L. Shaneson, Fixed points of periodic differentiable maps, Invent. Math. 68 (1982), $1-19$.

[6] E. C. CHо, Smith equivalent representations of generalized quaternion groups, Group actions on manifolds (Boulder, Colo., 1983), 317-322, Contemp. Math. 36, Amer. Math. Soc., Providence, RI, 1985.

[ 7 ] E. C. CHO AND D. Y. SUH, Induction in equivariant $K$-theory and $s$-Smith equivalence of representations, Group actions on manifolds (Boulder, Colo., 1983), 311-315, Contemp. Math. 36, Amer. Math. Soc., Providence, RI, 1985.

[ 8 ] J. H. Conway, R. T. Curtis, S. P. Norton, R. A. Parker And R. A. Wilson, Atlas of finite groups, Maximal subgroups and ordinary characters for simple groups, With computational assistance from J. G. Thackray, Oxford University Press, Eynsham, 1985.

[ 9 ] B. N. Cooperstein, Maximal subgroups of $G_{2}\left(2^{n}\right)$, J. Algebra 70 (1981), 23-36.

[10] K. H. Dovermann, Even-dimensional $s$-Smith equivalent representations, Algebraic topology, Aarhus 1982 (Aarhus, 1982), 587-602, Lecture Notes in Math. 1051, Springer, Berlin, 1984.

[11] K. H. Dovermann And M. Herzog, Gap conditions for representations of symmetric groups, J. Pure Appl. Algebra 119 (1997), 113-137.

[12] K. H. Dovermann And T. Petrie, Smith equivalence of representations for odd order cyclic groups, Topology 24 (1985), 283-305.

[13] K. H. Dovermann And D. Y. Suh, Smith equivalence for finite abelian groups, Pacific J. Math. 152 (1992), 41-78.

[14] K. H. Dovermann And L. C. Washington, Relations between cyclotomic units and Smith equivalence of representations, Topology 28 (1989), 81-89.

[15] The GAP Group, GAP-Groups, Algorithms, and Programming, Version 4.4.12, 2008.

[16] D. Gorenstein, R. Lyons And R. Solomon, The classification of the finite simple groups. Number 3. Part I. Chapter A, Almost simple $K$-groups, Math. Surveys Monogr. 40, American Mathematical Society, Providence, RI, 1998.

[17] P. B. KLeidman, The maximal subgroups of the Chevalley groups $G_{2}(q)$ with $q$ odd, the Ree groups ${ }^{2} G_{2}(q)$, and their automorphism groups, J. Algebra 117 (1988), 30-71.

[18] P. B. KLEIDMAN, The maximal subgroups of the Steinberg triality groups ${ }^{3} D_{4}(q)$ and of their automorphism groups, J. Algebra 115 (1988), 182-199.

[19] E. Laitinen And M. Morimoto, Finite groups with smooth one fixed point actions on spheres, Forum Math. 10 (1998), 479-520.

[20] E. Laitinen AND K. PAWAŁowsKi, Smith equivalence of representations for finite perfect groups, Proc. Amer. Math. Soc. 127 (1999), 297-307. 
[21] M. W. Liebeck And J. SAXL, On the orders of maximal subgroups of the finite exceptional groups of Lie type, Proc. London Math. Soc. (3) 55 (1987), 299-330.

[22] G. MALle, The maximal subgroups of ${ }^{2} F_{4}\left(q^{2}\right)$, J. Algebra 139 (1991), 52-69.

[23] M. Masuda And T. Petrie, Lectures on transformation groups and Smith equivalence, Group actions on manifolds (Boulder, Colo., 1983), 191-242, Contemp. Math. 36, Amer. Math. Soc., Providence, RI, 1985.

[24] J. MiLnOR, Whitehead torsion, Bull. Amer. Math. Soc. 72 (1966), 358-426.

[25] M. Morimoto, Smith equivalent $\operatorname{Aut}\left(A_{6}\right)$-representations are isomorphic, Proc. Amer. Math. Soc. 136 (2008), 3683-3688.

[26] M. MoRimoto, Nontrivial $\mathcal{P}(G)$-matched $\mathcal{S}$-related pairs for finite gap Oliver groups, J. Math. Soc. Japan 62 (2010), 623-647.

[27] M. Morimoto And K. PawaŁows Ki, Smooth actions of finite Oliver groups on spheres, Topology 42 (2003), 395-421.

[28] M. Morimoto And Y. QI, Study of the Smith sets of gap Oliver groups, Transformation groups from a new viewpoint (Kyoto, 2009), RIMS Kokyuroku 1670 (2009), 126-139.

[29] M. Morimoto And Y. QI, The primary Smith sets of finite Oliver groups, Group actions and homogeneous spaces, 61-73, Fak. Mat. Fyziky Inform. Univ. Komenského, Bratislava, 2010.

[30] M. Morimoto, T. Sumi And M. Yanagihara, Finite groups possessing gap modules, Geometry and topology: Aarhus (1998), 329-342, Contemp. Math. 258, Amer. Math. Soc., Providence, RI, 2000.

[31] B. OLIVER, Fixed point sets and tangent bundles of actions on disks and Euclidean spaces, Topology 35 (1996), 583-615.

[32] R. OLIVER, Fixed-point sets of group actions on finite acyclic complexes, Comment. Math. Helv. 50 (1975), 155-177.

[33] K. Pawalows Ki And R. Solomon, Smith equivalence and finite Oliver groups with Laitinen number 0 or 1, Algebr. Geom. Topol. 2 (2002), 843-895 (electronic).

[34] K. PawaŁows Ki And T. Sumi, The Laitinen conjecture for finite solvable Oliver groups, Proc. Amer. Math. Soc. 137 (2009), 2147-2156.

[35] K. PawaŁows Ki and T. Sumi, The Laitinen conjecture for finite non-solvable groups, Proc. Camb. Philo. Soc. 56 (2013), 303-336.

[36] T. Petrie, Three theorems in transformation groups, Algebraic topology, Aarhus 1978 (Proc. Sympos., Univ. Aarhus, Aarhus, 1978), 549-572, Lecture Notes in Math. 763, Springer, Berlin, 1979.

[37] T. PEtRIE, The equivariant $J$ homomorphism and Smith equivalence of representations, Current trends in algebraic topology, Part 2 (London, Ont., 1981), 223-233, CMS Conf. Proc. 2, Amer. Math. Soc., Providence, R.I., 1982.

[38] T. Petrie, Smith equivalence of representations, Math. Proc. Cambridge Philos. Soc. 94 (1983), 61-99.

[39] T. Petrie and J. Randall, Spherical isotropy representations, Inst. Hautes Études Sci. Publ. Math. 62 (1985), 221-256.

[40] T. Petrie AND J. Randall, Transformation groups on manifolds, Monographs and Textbooks in Pure and Applied Mathematics, vol. 82, Marcel Dekker Inc., New York, 1984.

[41] C. U. Sanchez, Actions of groups of odd order on compact, orientable manifolds, Proc. Amer. Math. Soc. 54 (1976), 445-448.

[42] A. W. Simpson And J. S. Frame, The character tables for SL(3, $q), \operatorname{SU}\left(3, q^{2}\right), \operatorname{PSL}(3, q), \operatorname{PSU}\left(3, q^{2}\right)$, Canad. J. Math. 25 (1973), 486-494.

[43] P. A. Sмiтh, New results and old problems in finite transformation groups, Bull. Amer. Math. Soc. 66 (1960), 401-415.

[44] R. Steinberg, The representations of $\operatorname{GL}(3, q), \operatorname{GL}(4, q), \operatorname{PGL}(3, q)$, and $\operatorname{PGL}(4, q)$, Canad. J. Math. 3 (1951), 225-235.

[45] D. Y. SUH, $s$-Smith equivalent representations of finite abelian groups, Group actions on manifolds (Boulder, Colo., 1993), 323-329, Contemp. Math. 36, Amer. Math. Soc., Providence, RI, 1985. 
[46] T. Sumi, Gap modules for direct product groups, J. Math. Soc. Japan 53 (2001), 975-990.

[47] T. SUmi, Gap modules for semidirect product groups, Kyushu J. Math. 58 (2004), 33-58.

[48] T. SUMI, Existence of a special $\mathcal{P}$-matched pair, Methods of transformation group theory (Kyoto, 2006), RIMS Kokyuroku 1517 (2006), 78-91.

[49] T. SUMI, Representation spaces fulfilling the gap hypothesis, Group actions and homogeneous spaces, 99-116, Fak. Mat. Fyziky Inform. Univ. Komenského, Bratislava, 2010.

[50] T. SUMI, The gap hypothesis for finite groups which have an abelian quotient group not of order a power of 2, J. Math. Soc. Japan 64 (2012), 91-106.

[51] T. SUMI, Smith sets of non-solvable groups whose nilquotients are cyclic groups of order 1, 2, or 3, RIMS Kôkyûroku Bessatsu B39 (2013), 149-165.

[52] T. Sumi, Centralizers of gap groups, Fund. Math. 226 (2014), 101-121.

[53] M. SUZUKI, On a class of doubly transitive groups, Ann. of Math. (2) 75 (1962), 105-145.

[54] K. B. TCHAKERIAN, The maximal subgroups of the Tits simple group, Pliska Stud. Math. Bulgar. 8 (1986), 85-93.

[55] R. A. Wilson, The geometry and maximal subgroups of the simple groups of A. Rudvalis and J. Tits, Proc. London Math. Soc. (3) 48 (1984), 533-563.

FACULTY OF ARTS AND SCIENCE

KYUSHU UNIVERSITY

744 MOTOOKA, NISHI-KU

FUKUOKA, 819-0395

JAPAN

E-mail address: sumi@artsci.kyushu-u.ac.jp 